\title{
Apparent size, apparent depth and the Müller-Lyer illusion
}

\author{
BARRIE STACEY ${ }^{1}$ AND RAYMOND PIKE ${ }^{2}$ \\ UNIVERSITY OF STRATHCL YDE, GLASGOW
}

The relationship between apparent size and apparent depth in certain forms of the Muller-Lyer illusion is explored both experimentally and theoretically. The experimental results show that the apparent sizes and apparent distances of the Muller-Lyer shafts in the normal and in the "reverse" illusions are related to each other. They also contradict specific predictions about the apparent depth location of Muller-Lyer shafts derived from the misapplied size-constancy theory and Emmert's Law. These results are interpreted in terms of the operation of the size-distance principle, and this interpretation is supported by observations of other illusions.

The present study investigates the relationship between apparent size and apparent depth in certain forms of the Müller-Lyer (M-L) illusion. Following a line of speculation by Thiery, Segall et al (1966) proposed that the shaft with outgoing fins is seen as longer than the shaft with ingoing fins, because it appears to be farther away. Pike and Stacey (1968) found that the shaft with outgoing fins tends to be seen as nearer than the shaft with ingoing fins when they are viewed as luminous figures under dark-room conditions. They suggested that the shaft with outgoing fins is seen as nearer because it appears to be longer, and that apparent depth may not be causally involved in the size distortion.

The misapplied size-constancy theory proposed by Gregory $(1963,1966,1968)$ is ambiguous concerning the relative depth location of the shafts in the usual M-L figures. Gregory simply concludes that his correlational evidence indicates that the shafts are independently distorted by the "perspective" of their attached fins without, for example, considering the possibility that the distortion may be responsible for the apparent "perspective" of the fins or that any apparent depth effect may be a consequence, rather than a cause, of the distortion.

The experiments of Yanasigawa (Oyama, 1960) and Fellows (1967) indicate that a continuous functional relationship exists between the illusion effect and the extent of the shafts. The usual M-L effect decreases to zero as the shafts decrease to fill about $7 / 10$ of the interfin space, and the "reverse" effect (i.e., the shaft between ingoing fins appearing longer) then increases until the shafts fill about half the interfin space, and then decreases to zero as the shaft lengths decrease to zero. Fellows $(1967,1968)$ proposed that the "reverse" M-L illusion is due to an expansion effect upon the shaft filling half the distance between the ingoing fins, caused by the enclosing nature of these fins, and that the reduction in the extent of the "reverse" illusion with increasing shaft length, and the eventual emergence of the normal illusion, are due to the progressive weakening of the enclosure effect and the strengthening of another effect specifically involving the pattern of the fins.

Stacey and Pike (1968), again using luminous figures in a dark room, examined the apparent depth location of shafts occupying half the interfin space in "reverse" M-L figures and found, for a majority of Ss, a reversal in apparent depth location as compared with the usual M-L figures. This finding is directly contrary to a prediction by Gregory (1967) derived from his illusion theory. He argued that a "half" shaft inside outgoing fins is displaced away from the ends of the bisecting fin lines towards the $O$ and shrunk by the size-constancy scaling mechanism. The implication for a "half" shaft between ingoing fins is clearly the opposite.

In view of the varying predictions of apparent depth in the M-L and incomplete M-L figures and the importance of these apparent depth features from a theoretical point of view, it is necessary to carry out further experiments that make a psychophysical comparison of parts of illusion figures in terms of apparent depth. Such experiments might also provide data that contribute to the evaluation of Fellows's enclosure hypothesis and make some small contribution to the resolution of the confusion in the literature concerning the experimental verification of the size-distance invariance hypotheses, and particularly Emmert's Law (Epstein et al, 1961; Fisher, 1968a; Gregory, 1966; Price, 1961).

\section{EXPERIMENT 1}

The experimental procedure used by Stacey and Pike (1968) did not totally control the sizes of the retinal images of the M-L shafts. Accordingly, it was decided to carry out an experiment in which the sizes of the retinal images of the shafts were always equated and that would also test a clear prediction from Fellows's (1967) experiment: that a reversal in the apparent depth location of shafts between ingoing and outgoing fin configurations would occur as the shaft length varied through $7 / 10$ of the interfin space.

\section{Apparatus}

Luminous $M-L$ fins, with each fin diagonal $4 \mathrm{in.}$ long and with an interfin distance of $10 \mathrm{in}$., were used. The left-right positions of the fins could be reversed. A pair of luminous shafts that filled 7/10 of the space between the ingoing and outgoing fins of a composite arrangement when placed adjacent to the fins were also used. The shafts could be moved as a pair to three positions behind the fins and three positions in front of the fins so that their retinal images filled approximately 0.49, 0.57, $0.63,0.70,0.76,0.84$, and 0.93 of the space between the ingoing and outgoing fins (Fisher, 1969). The Ss' view of each of the seven arrangements is shown in Fig. 1.

\section{Subjects}

Twenty-four adults who volun teered to cooperate in a study of depth perception were employed as Ss and paid a small sum.

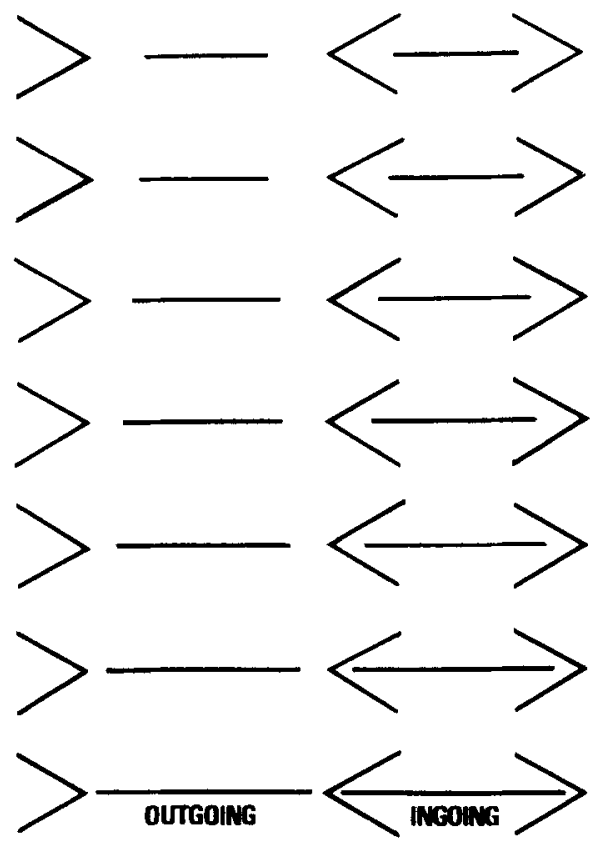

Fig. 1. The experimental arrangements of the $M-L$ fins. 


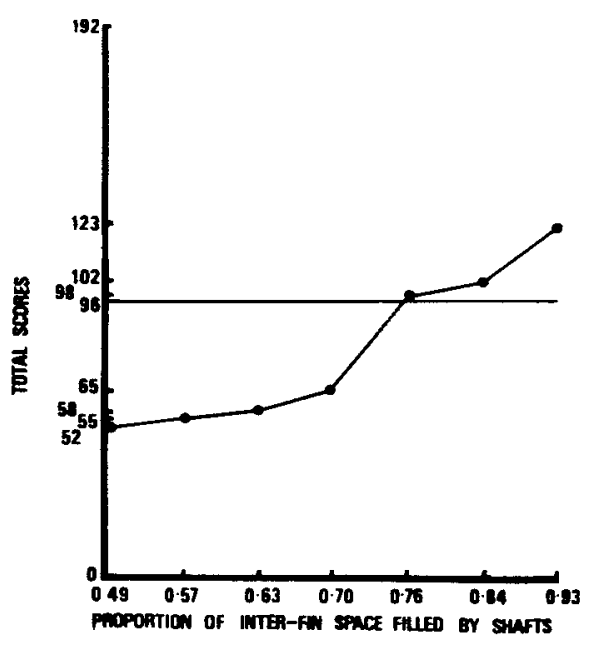

Fig. 2. Total scores for $24 \mathrm{Ss}$ in the distance judgment task.

They had no prior knowledge of the experiment or its rationale.

\section{Procedure}

The experiment was conducted in a dark room. The $S$ monocularly viewed the luminous fins at a distance of $10 \mathrm{ft}$ and the luminous shafts as a pair at distances of $13 \mathrm{ft} 6 \mathrm{in.,} 12 \mathrm{ft} 4$ in., $11 \mathrm{ft} 2$ in., $10 \mathrm{ft} 0$ in., $9 \mathrm{ft} 4$ in., $8 \mathrm{ft} 8 \mathrm{in}$., and $8 \mathrm{ft} 0 \mathrm{in}$. In each instance, he/she was instructed to say which of the two shafts, right or left, was the nearer. No limitation was placed on viewing time. For each of the seven shaft positions, eight judgments were made, four with the outgoing fins on the left and the ingoing fins on the right, and four vice versa. The judgments were made with the ordering of shaft positions randomized within and among Ss and fin conditions.

\section{Results}

The responses of the Ss were scored as +1 for "nearer" and 0 for "farther" for the shaft between outgoing fins. Each $S$ therefore could score from 0 to 8 for each shaft position. The overall scores may be read from Fig. 2, which gives the graph of the 24 Ss' scores against the proportion of the interfin space occupied by the shafts. A linear trend analysis of the scores is significant at the $p<.0001$ level. In terms of frequencies, 22 out of 24 Ss have higher scores on the three nearer positions combined as compared with the three farther positions combined, with one $S$ having equal scores. This distribution is significant at the $\mathrm{p}<.0001$ level (two-tailed binomial test). The effect of apparent size on apparent distance is well brought out by the results for the three farther positions where 23 of the 24 Ss have scores equal to or less than 9 (out of a maximum of 24), with an average score of 6.9 for all $24 \mathrm{Ss}$, whereas the null hypothesis specifies equal numbers on either side of a score of 12 . This distribution is significant at the $p<.0001$ level (two-tailed binomial test). For the three nearer positions, however, the null hypothesis would not be rejected.

\section{Discussion}

The most obvious result of this experiment is that a size-distance relationship has been demonstrated for apparent size when the retinal images of the judged stimuli are equal. It is true that no size-distance relationship is to be seen when the shaft lengths approach the usual M-L configuration. This may be partly the result of an increasing influence for accommodation and convergence as the distance of the shafts from the viewer is decreasing. However, the overall trend does indicate the presence of a continuous relationship.

\section{EXPERIMENT 2}

Fellows's enclosure hypothesis was supported by an experiment showing that a rectangle has a similar expansion effect upon shafts of varying length as ingoing fins. However, if enclosure is the crucial feature, then one would expect a completely enclosing rectangle to have a greater distorting influence than ingoing fins. Also, a rectangle surrounding a line could be interpreted as an interrupted extent illusion, as Fellows admits (1968, p. 371). Fisher (1968a) showed that a shaft bounded by an outgoing and an ingoing fin is not distorted. This arrangement appears to have enclosure properties midway between the completely ingoing and completely outgoing arrangements, and could be used with them to test the enclosure hypothesis. If an ingoing and outgoing fin configuration surrounds a "half" shaft, this enclosure should distort the perceived size of the shaft with consequences for its apparent depth location, placing it about midway between "half" shafts enclosed by completely ingoing and completely outgoing arrangements. With a "seven-tenths" shaft, there should be no size distortion with any of the three arrangements and, hence, no relative apparent depth effect.

\section{Apparatus}

Three pairs of luminous M-L fins of the same dimensions as in the previous experiment were used: one pair ingoing, one pair outgoing, and one pair with an ingoing and an outgoing fin (see Figs. 3 and 4). Two interfin distances were used, $10 \frac{1}{2}$ in. and 14 in. A luminous shaft, 7 in. long, was also used. The shaft could be moved in the horizontal plane in a direction along the viewer's line of regard by means of a pulley device.
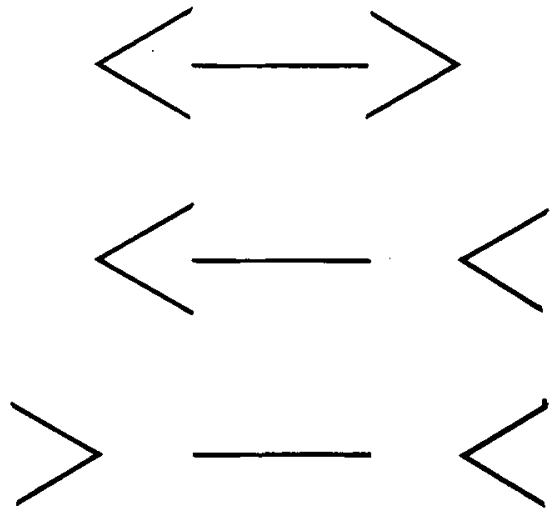

Fig. 3. The experimental arrangements of the enclosing fins for the half condition.

\section{Subjects}

Twenty-five adults who volunteered to cooperate in a study of perception were employed as Ss and paid a small sum. They had no prior knowledge of the experiment and had not taken part in the previous experiment.

\section{Procedure}

The experiment was carried out under dark-room conditions. The $S$ was presented with the shaft surrounded by one pair of fins at a distance of $10 \mathrm{ft}$. Using monocular vision, he/she was required to adjust the position of the shaft until it was the same distance away from him/her as the "corners of the two shapes." For each pair of fins, 10 adjustments were carried out by every $S, 5$ with the shaft filling half the interfin space and 5 with the shaft filling 7/10 of the interfin space. The adjustments were always made with the shaft in the same initial position, adjacent to the fins. The Ss' view of each of the six experimental arrangements is shown in Figs. 3 and 4. The ordering of arrangements was randomized within and
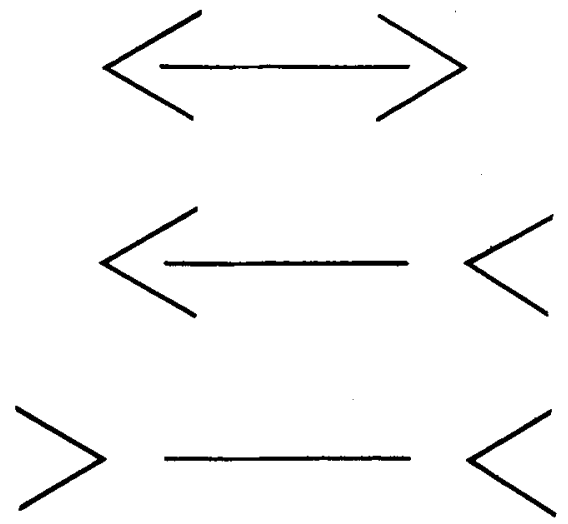

Fig. 4. The experimental arrangements of the enclosing fins for the $7 / 10$ condition. 
Table 1

Group Average Adjustment Errors

\begin{tabular}{|c|c|c|c|c|c|}
\hline \multicolumn{3}{|c|}{$\begin{array}{l}\text { Half Condition } \\
\text { Fin Characteristics }\end{array}$} & \multicolumn{3}{|c|}{$\begin{array}{c}\text { Seven-Tenths Condition } \\
\text { Fin Characteristics }\end{array}$} \\
\hline $\begin{array}{l}\text { Completely } \\
\text { Ingoing }\end{array}$ & $\begin{array}{l}\text { Ingoing and } \\
\text { Outgoing }\end{array}$ & $\begin{array}{l}\text { Completely } \\
\text { Outgoing }\end{array}$ & $\begin{array}{c}\text { Completely } \\
\text { Ingoing }\end{array}$ & $\begin{array}{l}\text { Ingoing and } \\
\text { Outgoing }\end{array}$ & $\begin{array}{c}\text { Completely } \\
\text { Outgoing }\end{array}$ \\
\hline $\begin{array}{l}+2.24 \text { in. } \\
\quad \text { a } \\
\text { Comparisons } \\
\text { Using t-Test }\end{array}$ & $\begin{array}{ccc}-0.90 & \text { in. } \\
& \text { b } \\
\text { a } & \text { vs } & \text { b } \\
\text { a } & \text { vs } & c \\
\text { b } & \text { vs } & \text { c } \\
\end{array}$ & $\begin{array}{l}-1.16 \text { in. } \\
\quad c \\
p<.01 \\
p<.01 \\
\text { n.s. }\end{array}$ & 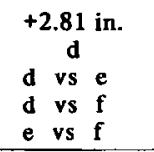 & $\begin{array}{l}+1.44 \text { in. } \\
\quad \text { e } \\
\text { n.s. } \\
\text { p }<.05 \\
\text { n.s. }\end{array}$ & $\underset{f}{+0.72}$ in \\
\hline
\end{tabular}

between Ss. The adjustment was experienced as a fairly difficult task, and no restriction was placed on vie wing time.

\section{Results}

For each $S$, the mean error of the five adjustments for each of the arrangements illustrated in Figs. 3 and 4 was calculated, yielding six mean error measurements. In an analysis of variance, only the interaction between the fin configurations and shaft lengths is significant $(F=3.93, d f=2 / 48$, $\mathrm{p}<.05)$. The main results are given in Table 1 , where a plus sign means that the group average adjustment error is beyond the fin configuration (i.e., away from the viewer), and a minus sign means it is in front of the fin configuration (i.e., towards the viewer). Individual comparisons between treatments indicate two significant differences between particular fin configurations when the shaft fills half the interfin distance and one significant difference when the shaft fills $7 / 10$ of the interfin distance.

\section{Discussion}

The results provide little support for Fellows's enclosure hypothesis, though they do not contradict it. In the half condition, the apparent depth location of the shaft between ingoing and outgoing fins falls, as predicted, between those of the completely ingoing and completely outgoing configurations. However, this apparent depth location is extremely close to that of the outgoing configuration and significantly distant from that of the ingoing configuration. In the $7 / 10$ condition, it appears that the completely ingoing configuration is exerting a distorting influence on the shaft affecting its apparent depth location. This, taken with the results of the previous experiment, suggests that the point of neutrality for apparent depth is not 0.70 of the interfin distance, but is approaching 0.76 . The $7 / 10$ condition operated as a weak second test of the enclosure hypothesis rather than as a control. In any event, it provided a confirmation of the trend in the half condition and an equivocal result as far as the enclosure hypothesis is concerned.
Compared with the enclosure characteristics of the completely outgoing configuration, those of ingoing and outgoing fins do not cause a distortion of a "half" shaft that significantly influences the estimation of its apparent distance. If there is enclosure-determined distortion of a "half" shaft between ingoing and outgoing fins, it is slight and certainly not half the distortion produced by a completely ingoing configuration. This suggests that whatever characteristic is the main cause of the reverse $\mathrm{M}-\mathrm{L}$ it is other than enclosure.

\section{CONCLUSIONS}

With perceived visual extent more or less constant, the perceived sizes and perceived distances of the M-L shafts in the usual and in the "reverse" conditions are related to each other. These experiential variables are associated in a lawful but seemingly complex way. Segall et al (1966) have argued that the M-L shafts (in the normal illusion) are seen as of different lengths because of a relative apparent distance effect between the two shafts, together with equality of retinal size of the shafts, the shaft "farther away" appearing longer. Gregory $(1966,1967,1968)$ has related the extent of the shaft distortion in a single M-L figure to its "perspective depth"; the farther away the shaft appears to be, the larger is its perceived size. Fisher (1968a) has forcibly argued that the apparent distances of the M-L shafts are irrelevant to an explanation of their differences in apparent size, and attempted an explanation of the relationship between apparent size and apparent depth in the M-L utilizing Emmert's Law. He came to the conclusion "that a line which appears longer also appears to be situated at a further distance than one which appears shorter." Fellows has argued for the operation of two distinct illusory forces in the usual and in the "reverse" M-L illusions, and avoided any consideration of the apparent depth features of the M-L figures. Is it possible to resolve at least part of this confusion?

The results of Experiments 1 and 2, and of the earlier experiments of the present writers, have contradicted specific predictions of Segall et al, Gregory, and
Fisher concerning the apparent distance locations of M-L shafts and, therefore, weakened their respective theoretical positions. The explanation of illusory distortion that proceeds to apparent size via apparent distance may be appropriate for geometrical illusions that contain unambiguous perspective or apparent depth information, but it appears to be inappropriate for the M-L. Gregory's claims that M.L figures display a "typical perspective view" and that all people spontaneously see luminous M-L figures three-dimensionally are simply erroneous (Fisher, 1968a; Hotopf, 1966; Pike \& Stacey, 1968). Further, Fisher (1968b, pp. 129-130) has experimentally demonstrated that varying the perspective features of composite M-L figures does not influence the magnitude of the illusion.

The explanation of the relative apparent distances of the M.L shafts in terms of Emmert's Law depends upon principles that were formulated to explain the apparent dimensions of projected afterimages in circumstances where information about physical distance is available. Artificial manipulation of distance cues can influence the perceived size of afterimages (Price, 1961). This explanation, assuming unambiguous distance information, also appears to be inappropriate for the M-L. Fisher's (1968a) analysis from apparent size to apparent distance seems to be inconsistent with his claim that there is no relationship between the magnitude of the M-L illusion and changes in the apparent distances of various shaft lengths (1968b, pp. 148-149).

If we consider the results of experiments utilizing luminous $\mathrm{M}-\mathrm{L}$ figures in dark-room conditions, they indicate that $S s$ judge the relative distances of the shafts in accordance with their apparent sizes. Because external depth cues are absent, and the retinal images of the shafts are equal or nearly equal, the apparently larger shaft is seen as nearer. These results are supported by observations that the moon looks both larger and closer on the horizon than at the zenith (Kaufman \& Rock, 1962) and that distant mountains appear bigger and closer than they actually are (Gibson \& Flock, 1962), and by the 
coffee cup illusion in which the image of a familiar object whose angular size increases as it moves closer to the viewer, is seen as being sharply diminished in size, and as moving away from the viewer (Senders, 1966). Also of relevance is the finding that an afterimage projected into the sky produces a "moon illusion" (Kammann, 1967; King \& Gruber, 1962). These results tend to support Fisher's general argument that the apparent distances of the M-L shafts are irrelevant as a causal explanation of why the shafts appear to differ in length. Taken with Fisher's experimental work, they strongly contradict Gregory's general argument that the magnitude of the illusion is determined by perspective depth. Finally, both Chiang's (1968) attempt to explain the M-L by diffraction of light and optical distortions of the eye, and Pressey's (1967) attempt on the basis of a central tendency effect that occurs in the judgment of magnitudes, clearly cannot explain the distortion phenomena of the usual and of the "reverse" M-L illusions.

\section{REFERENCES}

CHIANG, C. A new theory to explain geometrical illusions produced by crossing lines. Perception \& Psychophysics, 1968, 3, 174-176.

EPSTEIN, W., PARK, J., \& CASEY, A. The current status of the size-distance hypotheses. Psychological Bulletin, 1961, 58, 491-514.
FELLOWS, B. J. Reversal of the Muller-Lyer illusion with changes in the length of the inter-fins line. Quarterly Journal of Experimental Psychology, 1967, 19, 208-214.

FELLOWS, B. J. Reversal of the Müller-Lyer and "enclosure." British Joumal of Psychology, $1968,59,369372$.

FISHER, G. H. An experimental and theoretical appraisal of the inappropriate size-depth theories of illusions. British Joumal of Psychology, 1968a, 59, 373-382.

FISHER, G. H. The frameworks for perceptual localization Newcastle: Department of Psychology, University of Newcastle-Upon-Tyne, 1968b.

FISHER, G. H. Sizes of retinal images formed by distant objects. Nature, $1969,221,584-586$.

GIBSON, J. J., \& FLOCK, H. The apparent distance of mountains. American Joumal of Psychology, 1962, 75, 501-503.

GREGORY, R, L. Distortion of visual space as inappropriate constancy scaling. Nature, 1963, 199,678-680.

GREGORY, R. L. Eye and brain. London: Weidenfeld \& Nicolson, 1966.

GREGORY, R. L.Comments on the inappropriate constancy scaling theory of the illusions and its implications. Quarterly Journal of Experimental Psychology, 1967, 19, 219-223.

GREGORY, R. L. Visual illusions. Scientific American, 1968, 219, 66-76.

HOTOPF, W. H. N. The size-constancy theory of visual illusions. British Journal of Psychology, $1966,57,307-318$.

KAMMANN, R. The overestimation of vertical distance and slope and its role in the moon illusion. Perception \& Psychophysics, 1967, 2 585-589.
KAUFMAN, L., \& ROCK, I. The moon illusion. Scientific American, 1962, 207, 120-130.

KING, W. F., \& GRUBER, H. E. Moon illusion and Emmert's Law. Science, 1962, 135, 1125-1126.

OYAMA, T. Japanese studies on the so-called geometrical-optical illusions. Psychologia, $1960,3,7-20$.

PIKE, A. R., \& STACEY, B. G. The perception of luminous Muller-Lyer figures and its implications for the misapplied constancy theory. Life Sciences, 1968, 7, 355, 362 .

PRESSEY, A. W. A theory of the Müller-Lyer illusion. Perceptual \& Motor Skills, 1967, 25, 569-572.

PRICE, G. R. On Emmert's Law of apparent size. Psychological Record, 1961, 11, 145-151.

SEGALL, M. T., CAMPBELL, D. T., \& HERSKOVITS, M. J. The influence of culture on visual perception. New York: Bobbs Merrill, 1966.

SENDERS, J. W. The coffee cup illusion. American Journal of Psychology, 1966, 79, 143-145.

STACEY, B. G., \& PIKE, A. R. Depth location of shafts filling half the space between Muller-Lyer fins. Perceptual \& Motor Skills, 1968, 27 1019-1022.

NOTES

1. Address: Department of Psychology, University of Strathclyde, Glasgow, C1, Scotland. 2. Now at the University of Queensland.

(Accepted for publication May 13, 1969.) 